\title{
Raulin Anne, Les traces psychiques de la domination.
} Essai sur Kardiner

Marie-Antoinette Hily

\section{OpenEdition}

1 Journals

Édition électronique

URL : https://journals.openedition.org/remi/11106

DOI : $10.4000 /$ remi. 11106

ISSN : $1777-5418$

Éditeur

Université de Poitiers

Édition imprimée

Date de publication : 1 octobre 2018

Pagination : 339-341

ISBN : 979-10-90426-62-7

ISSN : 0765-0752

Référence électronique

Marie-Antoinette Hily, «Raulin Anne, Les traces psychiques de la domination. Essai sur Kardiner », Revue européenne des migrations internationales [En ligne], vol. 34 - n² et $3 \mid 2018$, mis en ligne le 28

décembre 2018, consulté le 16 avril 2022. URL : http://journals.openedition.org/remi/11106; DOI :

https://doi.org/10.4000/remi.11106 


\section{Notes de lecture}

Gallo, Ester

Scrinzi, Francesca

Migration, masculinities and reproductive

labour. Men of the home. - London, Palgrave Macmillan, 2016. - $311 \mathrm{p}$.

ISBN : 978-1-137-37977-1

De nos jours la figure de l'aide à domicile est une femme, très souvent immigrée. Ce métier fait partie de l'univers professionnel de l'aide et des services au domicile, très largement structuré par l'informalité et caractérisé par une pénibilité du travail et de faibles perspectives de mobilité sociale pour ses employé.e.s. Les analyses prospectives menées aux échelles nationale et internationale pointent la demande croissante d'aides à domicile liée au vieillissement de la population, mais l'incapacité des états à proposer un nouveau modèle économique et social, permettant d'améliorer la formation et les conditions de recrutement et de travail des personnels, contribue à la relégation de ces services à la périphérie du marché du travail et aux travailleurs les plus précaires. Une vingtaine d'années d'observations et d'enquêtes à travers l'Europe et l'Amérique du Nord a donné à voir divers mécanismes conduisant à une spécialisation du recrutement dans ces emplois et a nourri la théorisation du lien entre les rapports de genre et les métiers du care et aussi de l'articulation de ce lien avec les migrations. Comme tous les domaines identifiés comme " féminins " à cause de leur association avec des qualités dites naturellement féminines (l'écoute, le soin à autrui, etc.), ce domaine professionnel attire majoritairement des femmes (Perrot, 1987).

Le travail mené par Ester Gallo et Francesca Scrinzi prolonge cet effort collectif de recherches, en changeant de perspective. Elles ont pris pour objet de leurs investigations une figure moins visible du paysage du care : I'homme aide à domicile. Le contenu de ce livre s'appuie sur des recherches ethnographiques menées en Italie entre 1996 et 2012, des recherches menées sur le long terme auprès de migrant.e.s, employeurs, acteurs locaux (associatifs, religieux, syndicaux) et institutions politiques. Cette période a vu un foisonnement de recherches sur le contexte social, politique et économique de ce domaine professionnel, littérature à laquelle les deux chercheuses ont contribué. Les chapitres 2 et 3 présentent un riche état des lieux de cette littérature. Le deuxième chapitre offre une synthèse des travaux sur l'emploi des hommes migrants, notamment en Europe, avec une analyse de leur participation croissante aux emplois du secteur du care en tant que conséquence à la fois des politiques de migrations internationales et des régimes de protection sociale (portant sur le soin à domicile). Suite à la réduction de l'emploi dans des secteurs " masculins " comme le BTP, le rôle de la crise économique est aussi démontré. Quant au troisième chapitre, il passe en revue les perspectives féministes du racisme et de la racialisation spécifique des hommes et des femmes avec aussi une perspective tout à fait originale de la construction de la féminité racialisée en analysant comment la religion participe à la catégorisation des migrant.e.s sur le spectrum Nous/Autre. Le contexte italien du recrutement des aides à domicile est mis en exergue dans cette discussion autour de l'acceptation des femmes et des hommes - migrant.e.s travaillant au domicile des familles italiennes par les acteurs politiques ou religieux plutôt hostiles à l'immigration. 
La deuxième partie de l'ouvrage (les chapitres 4 à 7) s'articule autour de questions sur les modes d'entrée des hommes dans ces emplois, sur l'adaptation des hommes aux tâches représentées comme féminines et les effets de la masculinité dans les pratiques au travail, sur les relations de travail masculines employeur - employé, sur les impacts de l'expérience de ce métier sur la vie conjugale ou familiale des hommes, et enfin sur leur mobilité dans le secteur ou leur départ vers d'autres emplois. La richesse des analyses ne nous permet pas de tout mentionner ici. Nous allons pointer quelques-unes particulièrement originales.

D'autres études ont montré que l'entrée des hommes dans ces métiers se fait souvent grâce aux réseaux féminins, mais ce que révèle cet ouvrage est comment les pratiques des femmes conduisent à " domestiquer " l'identité masculine des hommes migrants, afin d'être conforme aux attentes des employeurs et de celles des acteurs impliqués dans la régularisation du statut sur le territoire italien (chapitre 4). Si on découvre comment les hommes aussi assurent un travail " émotionnel " auprès de leurs employeurs (les personnes âgées et leurs familles), la division sexuée du travail se recompose, car les hommes accèdent à des emplois plus variés et valorisants à leurs yeux. On voit également comment les hommes instrumentalisent des stéréotypes raciaux pour consolider leur position sur le marché du travail (et ce faisant, améliorer leurs chances de régularisation) : ils mobilisent de façon stratégique des constructions racialisées des aptitudes naturelles pour le métier en question. Dans le chapitre 5, la rencontre entre des masculinités hégémoniques (homme chef de foyer) et subordonnées (employé migrant) dans ce contexte est examinée selon le positionnement social de l'employeur, les plus aisés étant paternalistes, apportant de l'aide administratif à ces hommes migrants (proches aussi par la religion) pour la demande de régularisation tandis que pour les employeurs de milieux populaires, qui recrutent ces migrants par nécessité, les considèrent comme des concurrents. Mettant la focale dans le chapitre 6 sur l'expérience de ces hommes dans la vie conjugale et familiale, les auteures révèlent les impacts souvent complexes d'être le migrant qui suit la femme et de trouver un emploi avec I'" aide " des femmes et montrent des processus contradictoires de remise en question les hiérarchies genrées au sein du foyer et du renforcement ce celles-ci. Enfin, si la participation des hommes à ce domaine professionnel avait pu conduire à revaloriser le travail des femmes, les expériences des hommes rencontrés par les auteures du livre révèlent plutôt que la " respectabilité " obtenu par le travail dans le care, ainsi que d'autres acquis contribuant à un nouveau capital social (réseau de connaissances locales, savoir-faire auprès des institutions, langue) permettent aux hommes de sortir d'un emploi qu'ils considèrent toujours comme essentiellement féminin et accéder à des niches perçues comme spécialisées, masculines (gardien d'immeuble), sinon de sortir de la sphère privée vers d'autres métiers et étendre leur réseau de sociabilité masculine (chapitre 7).

Dans cet ouvrage, exercer le métier d'aide à domicile est analysé en tant que problème pour les hommes : être qualifiés pour y entrer, être respecté en tant qu'employé, " reconstruire leur masculinité ". La principale critique que I'on pourrait porter sur ce travail est le fait que l'expérience des hommes qui ne s'identifient pas avec des conduites et aspirations hétéro-normées est peu évoquée.

Tout en représentant une contribution importante à la littérature sur les migrations, ce travail apporte contribution significative au champ des études de genre. Jusqu'à présent, les travaux portant sur les " hommes dans les métiers féminins" concernent des environnements professionnels où ces hommes travaillent aux côtés de collègues femmes, permettant 
d'observer que souvent le simple attribut " masculin " positionne les hommes favorablement dans les relations de travail. Par la suite, ces hommes profiteraient d'un " escalator de verre "(Williams, 1992, citée dans Buscatto et Fusulier, 2013) qui faciliterait leur ascension sociale. Mais que se passe-t-il lorsque ces hommes sont des migrants ? Et qu'y a-t-il de spécifique dans les métiers qui s'exercent le plus souvent seul - au domicile de l'employeur ? Cet ouvrage montre encore une fois comment les rapports de genre jouent à l'intersection entre régimes du care et des migrations pour amener des personnes vers ce domaine professionnel, mais la grande originalité du travail de Ester Gallo et Francesca Scrinzi et de montrer comment l'expérience des hommes migrants aussi est marquée non seulement par les processus de racialisation, mais aussi par les rapports sociaux de genre.

\section{Références bibliographiques}

Buscatto Marie et Fusulier Bernard (2013) Présentation. Les " masculinités " à l'épreuve des métiers "féminins ", Recherches sociologiques et anthropologiques, 44 (2), pp. 1-19.

Perrot Michèle (1987) Qu'est-ce qu'un métier de femme, Le mouvement social, 140, pp. 3-8.

Stéphanie Condon

Chargée de recherche

INED

\section{Grenet, Mathieu}

La fabrique communautaire. Les Grecs à Venise, Livourne et Marseille 1770-1840. Rome : École française de Rome, École française d'Athènes, 2016. - $456 p$. ISBN : 978-2-7283-1210-8

L'ouvrage de Mathieu Grenet est bien davantage qu'une analyse particulièrement roborative de la présence grecque en Italie et en France durant la période 17701840. Issu d'une thèse de doctorat en histoire, il représente un jalon important dans le renouvellement historiographique des études portant sur les formes de groupement communautaires. Ne se satisfaisant pas de resituer dans leur contexte politique, socio-économique et religieux les modalités de cette présence pendant plus d'un demi-siècle, l'auteur met en lumière les multiples relations et échanges qui participent de cette fabrique communautaire et l'inscrivent plus largement dans une configuration diasporique.

Les observatoires choisis, Venise qui forme la colonie la plus ancienne, Livourne et Marseille, constituent les terrains propices pour saisir, à travers la morphologie socio-spatiale de leurs établissements respectifs, les dynamiques qui leur sont propres et leur maintien sur fond de changements. Certains s'énoncent d'ailleurs sous la forme de rupture comme la guerre d'indépendance et l'émergence d'un État-nation hellénique qui marquent la fin de la période considérée. Ce faisant, un intéressant problème est posé - qui ne manquera pas d'interpeler la communauté des chercheurs - sur la façon dont se pense le changement social au sein d'une configuration diasporique stable, façonnée par des interactions et des réseaux qui assurent la circulation des idées, des objets, des connaissances et des acteurs et structurent l'espace physique, social et symbolique de la diaspora.

La richesse de la perspective analytique choisie ne réside pas seulement dans la démarche qui consiste à remonter au général pour expliquer le particulier et inversement; elle tient surtout à une analyse fine des effets cognitifs induits par les outils descriptifs et discursifs habituellement mobilisés dans l'explication des faits sociaux historiques. Cette approche est étayée par des fonds archivistiques sélectionnés en tenant compte d'un souci d'équilibre qualitatif entre les trois colonies. On notera ainsi l'intérêt heuristique qui consiste à croiser les sources produites par les institutions grecques-orthodoxes avec 
celles émanant des trois municipalités étudiées ou encore, de façon plus inédite, avec la correspondance d'un intellectuel grec, Adamantios Koraïs.

Le fil d'Ariane de l'ouvrage posé en énigme - qui est Grec? - introduit la question des modalités d'élaboration pratique des différents marqueurs qui opèrent dans la construction relationnelle de cette appartenance. L'étude du cas grec devient alors particulièrement intéressante en raison de la mise en évidence d'un lent mouvement de définition de ce que serait une identité collective de référence autour de caractéristiques liées à des appartenances religieuse, linguistique et géographique et leur conversion en marqueurs ethniques, jalons significatifs d'un processus qui s'affirme de plus en plus, au fil de la période considérée, comme relevant du monopole des institutions communautaires. Ainsi, la densité de l'ouvrage tient tout autant à la façon dont sont saisies ces formes de présence communautaires - et on notera ici l'importance des repères visuels et sonores qui les inscrivent dans un espace (du) sensible - qu'à la façon dont elles participent plus largement de la "grécité» comme double principe de reconnaissance au sein du collectif de référence et de distinction à l'égard des autres. Ici réside I'un des apports les plus novateurs de l'ouvrage, car en enquêtant sur "la manière dont les acteurs historiques font communauté, ceci dans plusieurs environnements différents" (pp. 395-396), l'auteur met en évidence les différents modes opératoires par lesquels ce collectif rassemble et met en présence des acteurs paradoxalement unis par leur dépendance à l'égard d'une entité collective de référence.

Les analyses sont structurées en quatre parties comprenant deux chapitres chacune à l'exception de la dernière qui se termine par un épilogue. Les intitulés courts et explicites exposent les différentes facettes de ce fait communautaire grec au prisme des trois colonies étudiées. La première partie souligne ainsi explicitement dans son intitulé même "Présence", la façon dont la communauté se constitue comme telle au sein des sociétés d'accueil. II ne s'agit pas ici de réduire la diversité des profils qui s'offrent à l'analyse, ni du côté de la communauté grecque étudiée ni du côté de la société avec laquelle elle entretient d'ailleurs des rapports de contiguïté renouvelés. Le champ couvert par cette partie permet de mettre au jour des modalités inédites de présence, celle des femmes dans ces collectifs majoritairement masculins, celle des marins et des "gens de la mer" pris dans des parcours de mobilité ou encore celle de ces "passants ordinaires" qui, d'une colonie à I'autre, structurent les équilibres sociaux et démographiques en même temps qu'ils animent un réseau de relations dans et hors de l'Empire ottoman. La deuxième partie intitulée "Espace» porte sur les enjeux liés au processus de territorialisation de la communauté grecque à travers ses multiples dimensions, sociale, économique, symbolique et culturelle. L'espace est celui que dessinent les pratiques, notamment celles qui permettent de voir comment se distribuent les circulations. L'auteur analyse ainsi le processus de territorialisation qui opère à travers le fait de prendre place dans des territoires pris dans des hiérarchies locales et transnationales. La combinaison des différents dispositifs d'interaction participe ainsi de cette stabilisation du commun et contribue à l'insertion du local dans l'espace réticulé de la diaspora. Portant sur la fabrique du "lien communautaire», la troisième partie "Communauté" met en évidence le rôle des institutions dans la formation d'un sentiment commun d'appartenance dont la visée "performative» est de donner sens et consistance à la "grécité». Investies des fonctions religieuses, éducatives et caritatives, ces institutions organisent, contrôlent, représentent le groupe et le font exister comme entité sociale de référence à l'intérieur comme à l'extérieur du groupe. Enfin, la dernière partie intitulée 
«Recompositions" revient sur les deux événements majeurs de la période considérée, la guerre d'indépendance grecque et l'émergence du premier État national néohellénique, afin de souligner les effets de cette nouvelle "conscience nationale" sur la diaspora. Mettant à l'épreuve le postulat classique selon lequel l'expérience diasporique serait un laboratoire du national, l'auteur montre finement les lignes de tension qui traversent les colonies, des lignes de divergence révélatrices d'une identité en recomposition qui ne saurait se dissoudre mécaniquement dans la «nation».

Répondant à un double objectif d'analyse historique et contextualisée d'un fait communautaire et de mise en perspective critique des outils de la recherche, cet ouvrage vient non seulement combler un manque dans l'historiographie grecque à une période charnière, mais il représente également une contribution majeure à l'étude des formes communautaires de l'établissement humain. On ne saurait trop en recommander la lecture à l'heure où le fait communautaire est souvent présenté comme un modèle figé de présence, propice à l'essentialisation des groupes auxquels il s'applique. La fabrique communautaire rappelle en effet avec force que ce qu'il importe de connaitre ce n'est pas tant ce qui est produit que la façon dont cela l'est.

Constance De Gourcy

Sociologue, maître de conférences LAMES/Aix Marseille Université
Raulin, Anne

Les traces psychiques de la domination. Essai sur Kardiner. - Lormont : Le bord de I'eau, 2016. - $193 \mathrm{p}$.

ISBN : 978-2-35687-450-4

Dès les premières lignes de l'ouvrage qu'elle consacre à Kardiner ${ }^{1}$, Anne Raulin ${ }^{2}$ décrit I'homme comme psychanalyste, et cherche à aller au delà de celui qui fut connu comme l'initiateur du concept de " personnalité de base " et le contributeur du courant "Culture et personnalité ", aussi connu comme celui qui s'intéressait à l'histoire et à l'anthropologie, connu enfin comme celui qui dans la ville de Vienne des années 1920 rencontra Freud et écrivit en 1977 un remarquable récit de son analyse - "le roman d'un fils d'immigré très pauvre, que la misère étreint et humilie, et qui ignore longtemps ses propres ressources" (Kardiner, 1978 : 11). Si Kardiner a été ignoré par bien des anthropologues français, ce ne fut pas le cas du philosophe Merleau-Ponty qui dans ses cours à la Sorbonne (1949-1952) disait son intérêt pour l'anthropologie culturelle alors qu'il poursuivait l'objectif de travailler à une ontologie adéquate du collectif (Barthélémy, 2009 : 11).

La riche introduction d'Anne Raulin situe clairement son projet : remettre sur le métier la production d'une pensée et d'une œuvre qui avait ouvert en son temps une " route intellectuelle » de part et d'autre de

\footnotetext{
1 Abram Kardiner est né en 1891 à

Manhattan et mourut en 1981 dans le Connecticut. Son père quitta I'Ukraine pour I'Amérique en 1884. Sa femme le rejoignit par la suite avec leur fille aînée.

2 C'est l'intérêt pour The Mark of

Oppression écrit Anne Raulin (p. 35) qui va

la conduire à commencer son "enquête " sur "son auteur " au département de sociologie de Columbia University lequel a accueilli Kardiner et son projet d'étude sur la minorité africaine-américaine, pour I'achever au Schomburg Center for Research in Black Culture. Elle avait aussi rencontré la fille de Kardiner qui lui avait confié nombre d'archives concernant son père (voir Cheminements et remerciements, pp. 29-39).
} 
I'Atlantique. Le premier chapitre plonge le lecteur dans le rôle qu'a joué le premier conflit mondial sur les interrogations de Kardiner, qui travailla dès cette époque sur les traumatismes ou névroses de guerre ; dans les années 1930, son ouverture à l'anthropologie fut favorisé par le soutien des nombreux psychanalystes européens " réfugiés du nazisme " à NewYork (p. 25). Puis après la Seconde Guerre mondiale, le racisme accapara son attention dans une Amérique prenant conscience de la persistance des formes de ségrégation et de discrimination raciales. Ce livre permet ainsi de prendre la mesure de la richesse du milieu intellectuel ou la recherche de la vérité se mettait " au service des causes sociales et historiques " (p. 45).

Dans le second chapitre Anne Raulin s'attelle à comprendre l'homme Kardiner en prise avec son histoire (" travaillée par sa judéité " et " sa prédisposition à questionner la condition africaine-américaine dans ses paradoxes et ambivalences ": p. 26). Elle précise alors vouloir adopter une démarche chronotopique. Le chapitre trois est consacré aux démarches méthodologiques et aux collaborations scientifiques de Kardiner. Dans le chapitre quatre Anne Raulin s'efforce de dévoiler les raisons personnelles de la volonté de Kardiner d'être empirique ${ }^{3}$ dans ses démarches ; si la ville où il passa enfance et adolescence lui fournit une expérience de vie exceptionnelle, c'est à ses collaborateurs qu'il confiait en général les recherches empiriques (voir chapitre 3). Le dernier chapitre enfin renvoie à la ville encore comme espaces créateurs, comme espaces de brassages humains et intellectuels : dans les années 1940, ce n'était cependant plus la Harlem Renaissance et sa vitalité culturelle, et pas encore l'ère du mouvement des «Droits civiques, les mouvements

3 Kardiner pensait que la psychanalyse devait intégrer I'histoire sociologique des individus et créer ainsi un outil méthodologique heuristique (voir chapitre 4 " la marque de l'oppression "). nationalistes noirs et ceux de réafricanisation culturelle et religieuse» (p. 28), mais $c^{\prime}$ est là que fut conduite cette recherche sur "les traces psychiques de la domination" qui devaient éclairer sur la façon dont l'inconscient absorbe la discrimination raciale.

Arrêtons-nous sur le chapitre 3 et 4 où il est question de méthodes (les psychobiographies, "les études de personnalité») et d'exigence empirique. Les témoignages en situation (en contexte de face-à-face avec un médecin, le collaborateur de Kardiner : Lionel Ovesey) ont fourni les matériaux à The Mark of Oppression : réécrits sous forme de récits, ces entretiens sont classés selon des critères sociologiques, classe, genre et âge (lire des extraits, pp. 95-114). À la lecture de ces "comptes rendus" réalisés au début des années 1950, nous ne sommes pas si loin des entretiens que nous proposent les sociologues aujourd'hui dans la restitution de leurs recherches, mais sans l'«écoute empathique et psychanalytique" qui est l'essence même de la méthode psychobiographique où l'objectif thérapeutique est central : Kardiner "a le souci de la pratique, c'est-à-dire de la cure : il y a des hommes à guérir" écrit Mikel Dufrenne (p. 15). Le lecteur sera attentif à constater combien la couleur de la peau, la nature des cheveux, l'aspect corporel, la perception de soi sont pris comme critères de définitions des hiérarchies socio-raciales, etc. qui aujourd'hui relèvent de problématiques de la construction du rapport identité/altérité.

Une autre dimension des travaux de Kardiner retient l'intérêt, celle de la "formulation sociologique des émotions" (p. 119) où s'exprime "un monde hostile» et où les violences qu'elles viennent de l'intérieur du groupe ou de l'extérieur sont routines. Une "figure douloureuse" en prise à une forte émotion est emblématique, celle qui paradoxalement fige l'individu dans son appartenance raciale alors qu'il tend à vouloir " passer pour blanc " (p. 122) en s'élevant socialement par exemple. Dans 
la problématique de Kardiner, la violence des affects, les tiraillements entre la fidélité au groupe et le désir de s'en échapper pour une existence autre n'ont d'issue qu'au risque de devenir un traitre aux yeux des siens. Mais c'est aussi " ce sentiment de malaise, cette inadéquation psychique qui poussent des jeunes gens de classe moyenne à venir en psychothérapie, ou du moins à participer à cette enquête " (p. 123).

Si l'on s'attarde sur la question centrale de "la ligne de couleur ", on retiendra I'hommage que rend Anne Raulin à Kardiner, à ses collaborateurs et à ses collègues présents dans son séminaire, organisé dans les années 1930, que se tenir dans " la distanciation anthropologique " a évité la pente de l'interprétation de la condition noire en termes de pathologie sociale (p. 129) : " pour Kardiner et Ovesey, c'est la hiérarchie raciale et sociale au sein de la société occidentale qui est pathologique ", p. 130).

Kardiner est psychanalyste et il le reste dans tous les travaux qu'il a conduits et c'est à ce titre qu'il reconnaît « sa dette envers la sociologie africaine-américaine " et ses grands représentants, dont Frazier. Et c'est en tant que psychanalyste qu'il explore la " personnalité du Noir américain ", mais en s'emparant des expériences multiples de l'individu qui en face à face avec le psychanalyste est en demande d'aide. Kardiner s'implique d'autant que sa propre expérience soutient sa compréhension; l'explication se tenant dans l'extériorité comme espace des causes, du racisme, de la domination et de la ségrégation socioraciale. Et à quelles fins ce puissant investissement dans la recherche? Anne Raulin répond à cette question dans le chapitre 5 alors qu'elle mentionne la création de la Psychoanalytic and Psychosomatic Clinic for Training and Research que Kardiner fonde à cette époque à Columbia. À Harlem, d'autres initiatives aboutiront portées par des psychologues comme Kenneth Clark ou des écrivains comme
Richard Wright. L'auteure souligne aussi la place et le rôle qu'occupent les fondations privées, créées dans le but de développer les sciences sociales, dont la recherche sur les Africains-Américains.

"Rapprocher des idées que personne ne croyait compatibles " (p. 168), cette phrase de $\mathrm{C}$. Wright Mills vient à propos conclure cette note de lecture sur le bel essai que nous livre Anne Raulin. Mais on ne peut s'arrêter là. Et il faut lire la conclusion (" De l'histoire au présent ") pour regretter que l'auteure ne nous informe plus avant sur la réception de l'œuvre de Kardiner et de son relativisme culturel, qui a contribué à la lutte contre les préjugés racistes et sexistes, chez les intellectuels français.

II convient de saluer l'entreprise d'Anne Raulin qui arrache l'œuvre de Kardiner du sommeil et la sensibilité de l'auteure à I'histoire de l'anthropologie culturelle américaine. Elle rappelle ainsi à celles et ceux qui l'ont oublié la place qu'a occupée la psychanalyse dans le projet de compréhension des sociétés, démarche qui ne participe plus guère aujourd'hui au projet d'interdisciplinarité de la recherche contemporaine sur les migrations.

On retiendra de ce travail, bien documenté, l'effort de l'auteure pour faire revivre la pensée de Kardiner dans sa volonté de saisir les objets de recherche sous différents angles disciplinaires ce que résumait Bastide par : " action et réaction constantes du social sur le psychique et du psychique sur le social " (voir note 5 p. 17).

\section{Références bibliographiques}

Barthélémy Jean-Hugues (Dir.) (2009) Cahiers Simondo, n¹, Paris, L’Harmattan, $156 \mathrm{p}$.

Kardiner Abram (1978) Mon analyse avec Freud, Préface de Mikel Dufrenne, Paris, Belfond, $136 \mathrm{p}$.

Marie-Antoinette Hily Migrinter/CNRS 
Cerrato Debenedetti, Marie-Christine

La lutte contre les discriminations ethnoraciales en France. De l'annonce à l'esquive (1998-2016). - Rennes : PUR, 2018. - 255 p. ISBN : 978-2-7535-2-7323-9

L'ouvrage de Cerrato Debenedetti nous livre une analyse passionnante et sans concession de la politique française de lutte contre les discriminations ethnoraciales initiée en 1998 par gouvernement de Lionel Jospin. Le tabou sur les discriminations raciales ayant été levé, ce projet initial "audacieux» portait en lui une double promesse : transformer les rapports globaux entre les immigrés et la société et reconnaître, à la fois, la responsabilité de la société dans la production des discriminations ethno-raciales et leur impact sur le processus d'intégration sociale. Cependant, les résultats modestes de cette politique menée entre 1998 et 2016, marquée par de nombreuses "hésitations", "esquives" voire "effacements", conduit plutôt au désenchantement : la lutte de l'État contre les discriminations ethno-raciales, constamment déniées ou euphémisées, est une politique du statu quo qui n'a pas rendu ces discriminations suffisamment "ignobles" pour réussir à élaborer une culture du problème et une croyance en leur possible éradication.

L'ouvrage très dense est composé de six chapitres. Les trois premiers fournissent de nombreuses informations et clefs de compréhension des logiques de I'action publique et du faible engagement de l'État dans la reconnaissance et le traitement des discriminations ethno-raciales. L'auteure révèle les postures politiques de déni de ces discriminations, les multiples arguments des dénégateurs, y compris institutionnels, et leurs rôles dans leur coproduction. La territorialisation de cette politique en 2001et son inscription dans la politique de la ville (voire sa mise en concurrence avec elle) camouflent une triple esquive : "esquive de la définition et de la prise en charge nationale du problème par la territorialisation, esquive de la question ethnique par le territoire et enfin esquive du débat politique par les instruments" (p. 112). Cette "délocalisation" ne témoigne pas d'une volonté de généraliser la politique de lutte contre les discriminations ethno-raciales à tous les territoires, mais révèle davantage le désintérêt et l'absence d'engagement de l'État qui contribuent à son affaiblissement.

Celui-ci se poursuit et s'accentue avec I'universalisation des discriminations reconnues par les directives européennes puis avec la politique de diversité/d'égalité des chances qui déclasse voire efface les discriminations ethno-raciales au bénéfice des discriminations de sexe, d'âge et de handicap. Par ailleurs, I'auteure note que la production de nombreux outils de mesure, de formations et de bonnes pratiques (chartes et labels) reproductibles ou transférables, sous l'impulsion du programme EQUAL, met davantage en valeur l'action que les discriminations ethno-raciales. Enfin, elle souligne que la recomposition du champ de l'expertise et des professionnels de l'intervention sociale et les enjeux de (dé)légitimation professionnelle et de technicisation de l'action participent aussi de la marginalisation des discriminations ethno-raciales et que l'absence de mobilisation citoyenne contre ces discriminations a contribué à l'orientation mineure de la politique.

Les chapitres 4,5 et 6 apportent de nombreux éléments de connaissance empirique basée sur une recherche doctorale initiée en 2006 à Sarly; nom d'emprunt d'une ville où, par contraste avec le "faible portage» de la politique de I'État, la mobilisation politique, institutionnelle et citoyenne de lutte contre les discriminations ethno-raciales est aussi exceptionnelle que fragile. En cela, Sarly s'apparente au dernier village gaulois constamment menacé par les légions romaines tant les résistances à la reconnaissance et à la prise en charge de ces discriminations sont tenaces. 
Ces chapitres rendent minutieusement compte du processus de construction collective de ce que Conan (1998) nomme "un souci commun» par les acteurs concernés (élus, représentants d'associations, citoyens et professionnels) pour définir un problème et les modalités de l'action publique, à partir des différents points de vue. Debenedetti décrit les acteurs impliqués et analyse les stratégies et les dispositifs mis en place à Sarly ${ }^{4}$ pour créer une culture du problème des discriminations ethno-raciales et une dynamique locale autour de cette question. L'originalité de la démarche, son défi même, réside dans la reconnaissance de la responsabilité des acteurs institutionnels et des intermédiaires (de l'emploi, scolaire ou du logement) dans la coproduction des discriminations qui nécessite d'envisager dans la stratégie d'action les discriminés et des discriminateurs. Ce qui ne se fera pas sans résistances de la part des seconds.

La stratégie locale de Sarly s'est appuyée, d'une part, sur la mobilisation du droit comme norme et outil pédagogique d'aide à la définition et à la prise de conscience des contours des discriminations ethno-raciales. L'auteure révèle que, contrairement à d'autres législations, le droit de la non-discrimination ne fait pas autorité ni consensus dans la population : il est souvent discuté et remis en cause par les discriminateurs et mis en concurrence avec les valeurs de la République, la laïcité ou la protection de l'ordre public. D'autre part, la ville a créé un dispositif participatif d'aide à l'expression des expériences de la discrimination, "La discrimination, parlons-en?», qui ambitionnait de transformer l'expérience de l'injustice des discriminés en revendication et actions politiques. L'analyse met en relief les impacts individuels et collectifs de l'expérience

4 L'auteure y occupait un poste de chef de projet sur la lutte contre les discriminations qui lui a donné un accès privilégié aux acteurs locaux concernés par le problème. des discriminations qui éclaire les raisons de l'absence de mobilisation des discriminés pour les combattre et le mutisme à leur sujet : la crainte d'être pris pour un mythomane et le coût psychologique et social d'une mise en récit du vécu des discriminations ethno-raciales. L'auteure montre que le "cheminement" cognitif et subjectif des discriminés pour reconnaître puis dénoncer les discriminations transforme progressivement leur posture en sujet de droit, mais ne débouche pas sur l'engagement politique espéré : il convertit l'expérience personnelle en un "concernement» sans engagement militant.

La réussite exemplaire de la politique de Sarly à mesurer la réalité des discriminations ethno-raciales, à les faire reconnaître publiquement et à élaborer une action publique tient à la conjonction d'occurrences singulières : l'engagement déterminé d'un adjoint au maire et du maire de la commune en dépit du déni et de délégitimation tenaces de ces discriminations à l'échelle nationale et locale, la création d'espace de légitimation des actions (une mission de lutte contre les discriminations, la commission de lutte contre les discriminations ethniques, le réseau de vigilance et de lutte contre les discriminations, et l'observatoire des discriminations), et le concours des partenaires locaux, des experts formateurs, des chercheurs et les "propriétaires" du problème (FASILD, HALDE puis Défenseur des droits). Debenedetti reconnait cependant qu'elle repose sur un équilibre précaire lié à des personnalités très concernées et qu'elle pourrait s'affaiblir voire s'effacer avec leur départ.

L'analyse stimulante de la politique de lutte contre les discriminations ethnoraciales a une portée qui dépasse les frontières de la France. Les constats établis sont, à bien des égards, analogues à ceux observés dans d'autres pays européens comme la marginalisation des discriminations ethno-raciales par leur universalisation, la politique de promotion de 
la diversité et la technicisation des outils et des pratiques; la dépolitisation et les nombreuses résistances au droit, et la faible mobilisation citoyenne sur cette question. Cette étude, qui fera date, apporte des éclairages indispensables à la compréhension du bilan pessimiste de près de deux décennies d'une politique antidiscriminatoire envers les minorités ethno-raciales que l'Agence européenne des Droits Fondamentaux a révélé dans sa dernière enquête (FRA, 2017) qui indique que ces populations restent les plus discriminées et les plus vulnérables de I'Union européenne.

\section{Références bibliographiques}

Conan Michel (1998) L'évaluation constructive. Théorie, principes et éléments de méthode, Paris, Éditions de l'Aube, $196 \mathrm{p}$.

FRA (2017) Second European Union Minorities and Discrimination Survey (EU MIDIS II), Vienna, EU Fundamental rights Agency, [online]. URL: http://fra.europa.eu/en/publication/2017/eumidis-ii-main-results

Nouria Ouali

Sociologue, Professeure METICES/Université Libre de Bruxelles

\section{Wang, Simeng \\ Illusions et souffrances. Les migrants chinois à Paris. - Paris : éditions Rue d'Ulm sciences sociales, 2017. $-220 \mathrm{p}$. \\ ISBN : 978-2-7288-0574-7}

Simeng Wang, sociologue, jeune chercheure au CNRS nous livre un ouvrage de 218 pages, décomposé en une introduction, six chapitres, une conclusion, des notes par chapitre, une bibliographie, une bibliographie en chinois. Simeng Wang nous livre une sociogenèse originale des souffrances des migrants chinois à Paris à travers le prisme de leurs accès aux soins psychiatriques, de l'appropriation et des usages qu'ils ont d'une "culture psy». Ce travail a pour objectif d'offrir des connaissances sociologiques sur les Chinois de Paris et de complexifier le regard trop souvent réducteur porté sur cette communauté. II s'agit d'analyser les souffrances psychiques de ces migrants et leur recours à une prise en charge que celle-ci relève de la psychiatrie, psychologie, psychothérapie ou psychanalyse. Simeng Wang met en lumière les multiples souffrances qui affectent les migrants chinois et leurs descendants à travers les difficultés qu'ils traversent au fil de leurs parcours migratoires, de leur installation et leur vie en France dans une perspective d'ascension sociale.

Une place privilégiée est accordée à la question de la stratification sociale telle qu'elle est définie en Chine et mise à l'épreuve par l'expérience transnationale, l'auteure entraîne ainsi le lecteur dans l'intimité des familles chinoises à Paris dessinant la pluralité des trajectoires et des configurations, et signifiant l'importance des contextes en Chine et en France. L'appartenance sociale (conscience d'être d'un certain milieu, mobilité sociale, stratégie de distinction, habitus, transmission de la mémoire, le rôle de la parenté) conditionne les parcours des individus, les problèmes auxquels ils se heurtent, les souffrances les étreignent et leur capacité de résilience. On sent que cette question de la stratification sociale est sensible à la fois pour l'auteure lorsqu'elle se livre à un détour réflexif pudique et pour les personnes chinoises qui participent à son enquête : chacun à sa manière exprime ses origines, ses relations avec ses parents, sa position dans le récit familial, ce qui le distingue d'autres catégories de migrants chinois. Migrants venus d'une société marquée par l'absence relative de classes sociales, la prédominance du nous collectif surtout avant les années 1980, le manque de possibilités d'expression personnelle, la vie en Occident leur révèlent à quel point ils sont les acteurs de leur migration.

Dans sa partie introductive, l'auteure explicite la construction de son objet, sa démarche méthodologique au sein d'espaces familiaux et thérapeutiques 
dans différents arrondissements parisiens. L'écriture est vive, alerte, mesurée, se refusant à toute généralisation. Le premier chapitre restitue les contextes d'émigration et d'immigration en instant sur deux dimensions : l'évolution de la stratification sociale en Chine et les représentations de l'Occident en Chine et chez les migrants. Avec l'accession au pouvoir du parti communiste, la bourgeoisie disparaît et de nouvelles formes de distinctions sociales apparaissent. Durant la révolution culturelle, la mobilité sociale est déterminée par le lieu de naissance et la proximité avec la classe populaire, ces deux facteurs déterminants l'accès à l'éducation, au logement et au travail. À partir de la fin des années 1970, les changements politiques et économiques affectent ce déterminisme sociopolitique. L'accès et les possibilités de mobilité vont dès lors dépendre du capital social (guanxi) qui se définit comme le réseau ou l'entregent dont dispose l'individu. L'émigration chinoise vers la France est décomposée en trois vagues migratoires, mais tous les migrants impliqués dans ces flux n'ont pas fait l'objet de la même attention de la part des chercheurs. Simeng Wang souligne le manque de travaux relatifs aux migrants qualifiés alors qu'ils sont de plus en plus nombreux, ils ont été oubliés au profit de groupes plus visibles et devenus représentatifs de "la" communauté chinoise (les migrants non qualifiés originaires du Wenzhou et les Dongbeis arrivés clandestinement en France), et elle note l'absence de recherches portant sur les secondes générations chinoises comparativement à d'autres flux migratoires.

Le chapitre 2 s'attache à rendre compte de la production de la parole des réfugiés politiques installés en France suite aux événements de Tien'anmen et de leur répression. Simeng Wang revient en premier sur le contexte sociopolitique d'émigration et sur celui d'immigration favorable à l'accueil des jeunes intellectuels fuyant la Chine. Néanmoins il convient de distinguer deux situations, celle des leaders du mouvement de contestation qui furent contraints de quitter le pays et sont dans l'impossibilité d'y retourner et la situation d'autres jeunes libres de circuler désireux d'un nouveau cadre de vie et de pensée. L'auteure s'intéressant à la première catégorie rend compte du paradoxe de l'exil "comment le sentiment de n'être ni d'ici ni d'ailleurs s'articule avec l'engagement politique et les souffrances psychiques". Les réfugiés expriment la complexité des liens politiques, intellectuels et affectifs avec leur société natale et les discordances que la vie en exil génère (naturalisation, chinese feeling, l'amour paradoxal du pays natal, la pratique de la langue, culture intellectuelle). La difficulté et la douleur à parler de son expérience singulière se traduisent par la mise en retrait de la subjectivité au profit d'un récit plus englobant exprimant les souffrances de cette génération.

Se succèdent ensuite le chapitre 2 consacré aux réfugiés chinois ayant vécu les événements de Tian'amnen, le chapitre 3 aux jeunes migrants qualifiés ayant choisi de rester en France, le chapitre 4 aux migrants en situation irrégulière, le chapitre 5 aux descendants de migrants dits de première génération et demie, le chapitre 6 clôt ce panorama sociologique par les adolescents français d'origine chinoise. Ce dévoilement sensible de l'intimité des familles et des replis des âmes souffrantes de ces migrants offre une peinture subtile de ces communautés chinoises et tisse la trame d'un livre qui happe le lecteur. À travers la mise en intrigue réussie des récits des migrants et de leurs descendants grâce à l'intrication constante d'éléments de contextualisation et d'extraits de verbatim, le lecteur est confronté aux problèmes quotidiens de ces migrants, qu'il s'agisse des difficultés à être régularisé, à réussir ses études, à vivre dans un logement réduit, à obtenir un emploi à la hauteur, à réussir son mariage, à trouver sa place entre les attentes structurantes de sa famille et le désir d'être soi. La confrontation entre des modèles 
familiaux chinois, d'autres modèles plus ou moins intégrés à la société française voire à l'espace mondialisé et la quête existentielle des jeunes générations qu'elles soient nées en Chine ou en France est passionnante.

Si l'ensemble des chapitres apporte des réflexions stimulantes, certains plus novateurs invitent à d'ultérieurs approfondissements. Évoquons la question de "l'incompréhension douloureuse" entre les enfants de migrants nés et restés en Chine et leurs parents qu'ils rejoignent des années plus tard en France. Ces enfants sont mis à contribution par leurs parents à travers des "obligations familiales à rebours", ces injonctions parentales sont douloureusement ressenties par ces jeunes, car ils ont le sentiment d'avoir été oubliés, de n'avoir pas reçu d'affection, d'avoir été instrumentalisés au profit d'autres réalisations familiales en particulier en milieu wenzhou. Cette carence émotionnelle engendre des relations filiales marquées par l'ambivalence qui conduisent ces jeunes à s'éloigner d'une vie dénuée des échanges affectifs et symboliques attendus. Cette distanciation est médiatisée par des institutions extérieures et la prise en charge psychiatrique permet parfois à ces jeunes d'échapper à ces obligations et de se recentrer sur leur propre devenir. La question de la gestion des souffrances psychiques liées aux aspirations sociales très encadrées des descendants de migrants chinois nés en France pose de multiples questions. Cette recherche exemplaire montre, s'il était encore besoin qu'il existe une pluralité de modes d'insertion dans la société française et que chaque migrant "fait France" de manière singulière.

Véronique Petit

Démographe, Professeure CEPED/Université Paris Descartes
Salmon, Jean-Marc

29 jours de révolution. Histoire du soulèvement tunisien, 17 décembre 2010-14 janvier 2011. - Paris : Les petits matins, 2016. -349 p. ISBN : 978-2-36383-198-9

S'attacher à décrire des faits contemporains maintes fois commentés par les médias et la sphère politique n'est pas chose aisée. C'est pourtant ce à quoi Jean-Marc Salmon s'est attelé dans cet ouvrage sur la révolution tunisienne de 2010-2011. L'ouvrage s'attache à la dimension historique des évènements et le sociologue commence d'ailleurs en introduisant par ces mots : "La première révolution du XXle siècle s'est produite en Tunisie» (p. 11) mettant d'emblée le lecteur face à la portée historique des faits, en les situant dans le cours du temps. Seulement cinq ans après, peut-on avoir assez de recul sur ces évènements? C'est la question que le lecteur peut se poser après la lecture de l'ouvrage. Cependant, $c^{\prime}$ 'est bien à partir d'une étude approfondie, constituée notamment d'entretiens avec près de quatre-vingt-dix acteurs de la révolution, que l'auteur montre à la fois la part d'improvisation du soulèvement tunisien, son caractère moderne et novateur par l'apport des nouvelles technologies et des réseaux sociaux, ainsi que sa rapidité. Le titre souligne bien ce dernier aspect : il aura fallu seulement vingt-neuf jours pour faire tomber un gouvernement installé depuis plus de vingt-trois ans.

Si les conséquences de ces évènements sont bien connues par leur importante médiatisation, le détail de ces vingt-neuf jours et leurs causes dans la société tunisienne sont moins connus. À la fois chronologie commentée et bilan des évènements, la description faite par Jean-Marc Salmon, à travers de ces dix-neuf chapitres, donne une analyse plus poussée sur les origines et le déroulement de la révolution. 
Une première partie est consacrée à ce que l'auteur appelle "l'underground des révoltes" et s'attache à revenir sur les prémices de la révolution. Elle aborde notamment l'un des aspects novateurs des évènements tunisiens : l'influence des médias et des réseaux sociaux. L'importance de la "cyberdissidence» à partir de blogs est évoquée, puis celle de Facebook à partir de 2008, comme vecteur important de transmission de l'information et de critique du pouvoir. Dans le même ordre d'idée, les révélations de scandales et corruptions du pouvoir par Wikileaks ont contribué à désacraliser le pouvoir permettant un affrontement dans la rue. Puis, l'auteur s'attarde en première, puis en deuxième partie sur la ville de Sidi Bouzid et le déclenchement de la révolte le 17 décembre 2010 où l'immolation du marchand Mohamed Bouazizi a suscité la colère de la population envers les autorités locales abusant de leurs privilèges. "Le suicide du pauvre» selon les termes de l'auteur concerne près de 200 Tunisiens par an (car l'essence bon marché est facile à se procurer), mais là où l'acte est exceptionnel, c'est qu'il se déroule en public et devant le siège du pouvoir local, le désignant ainsi implicitement comme fautif et faisant le lien avec les abus de la police locale qui avait confisqué les denrées et la charrette du marchand. Sans succomber au registre dramaturgique, l'auteur met en avant l'émotion suscitée par l'acte suicidaire et qui a engendré un rassemblement spontané qui déclenchera une action organisée : la foule se donne de la force et on fait du marchand le martyr d'un système corrompu dont les plus pauvres sont exclus. Les canaux d'informations et de communication tels que Facebook, France 24 ou Al-Jazira font la suite en diffusant l'information.

C'est ensuite avec minutie que l'auteur démontre l'effet "boule de neige" qui a permis, après la mort d'un second homme dont l'acte est également compris comme un suicide, l'extension du mouvement social aux villes voisines. Cyberactivistes, syndicalistes de gauche et avocats progressistes se mobilisent et le pari de manifester dans la capitale réussit. Les manifestations deviennent rapidement générales et ce sont les lycéens qui prennent rapidement le relais à la rentrée de janvier, notamment à Thala, petite ville de l'est du pays. La situation s'envenime vite jusqu'aux tirs de balles réelles par les forces de l'ordre et se propage alors au chef-lieu régional : Kasserine.Avec neuf morts, le bilan estlourd et n'apaise pas la situation. Au contraire, les insurgés en arrivent à l'«acceptation à mourir" (p. 216) qui explique en partie persévérance à manifester. De plus, la diffusion d'images et de vidéos dévoilant la violence des répressions fait monter la colère dans les milieux populaires de la côte et notamment de Tunis. On passe alors à une phase de nationalisation du "mouvement». Sfax, deuxième agglomération du pays, soutient très rapidement le mouvement, notamment car la ville est peuplée de nombreuses populations originaires de Sidi Bouzid. Entre défilés en journée et manifestations plus violentes la nuit et avec I'UGTT ${ }^{5}$ qui appelle à la grève générale, les opposants politiques au régime commencent à croire possible une chute du régime de Ben Ali, notamment quand ce dernier adoucit son discours face aux manifestants.

Au fur et à mesure des chapitres, on suit donc la révolution en train de s'organiser et à l'aide d'une analyse des réactions des médias et du pouvoir politique en place, Jean-Marc Salmon décrit la fin du régime de Ben Ali jusqu'à la fatidique journée du 14 janvier 2011 où le président démissionne. Là, c'est heure par heure qu'il nous livre le récit d'une journée qui a commencé par un nouvel appel à manifester de I'UGTT et qui abouti à la fuite du président et cette annonce de l'Agence France-Presse : "ALERTE (18 h 35). Le

5 L'Union générale tunisienne du Travail, première force syndicale du pays. 
président Ben Ali a quitté laTunisie (sources proches du gouvernement)». Enfin, la dernière partie se consacre aux aspects "inédits du soulèvement tunisien». Nous retenons notamment la tension dans l'usage des médias numérisés, entre une logique de contrôle du pouvoir face à un usage important des réseaux sociaux et médias dans ce soulèvement. De plus, le pouvoir s'est trouvé affaibli en perdant le monopole de l'image avec des chaînes arabophones transnationales et la perte de l'oral avec l'importance du langage dialectal (derja) sur les réseaux sociaux. L'image domine sur l'écrit dans cette révolution, alors même que l'écrit était un des fondamentaux du principe révolutionnaire comme le rappelle l'auteur. Cela montre la spontanéité du mouvement, sans texte fondateur, sans direction, sans porteparole, mais qui en vingt-neuf jours a fait fuir le président.

L'analyse détaillée des événements et les entretiens menés directement par l'auteur auprès des acteurs de cette révolution donnent ainsi une légitimité scientifique à la recherche entreprise par le sociologue. Les entretiens mettent par ailleurs en avant l'importance de la mémoire, encore vive aujourd'hui. Mais l'importance mémorielle laisse aussi place à la concurrence mémorielle et montre l'importance de croiser les données et de les diversifier. En effet, les évènements n'ont que cinq ans lorsque l'auteur entreprend son enquête, la compétition entre les acteurs face à la mémoire des évènements est déjà à l'œuvre. C'est là que le travail de complément d'analyse à partir de données socio-économiques trouve un sens et que la trame de la révolution et de ses prémices est suivie. On découvre ou redécouvre les fondements d'une contestation qui a beaucoup fait parler, beaucoup fait écrire, mais dont nous avons avec cet ouvrage une analyse solide, basée sur des faits concrets. L'auteur apporte les éléments permettant d'échapper à une lecture "héroïsée» des événements qui serait liée à une surenchère mémorielle face à des faits contemporains qui ont marqué les esprits.

Jordan Pinel

Géographe, Doctorant Migrinter/Université de Poitiers

Magri, Susanna (Dir.)

Tissot, Sylvie (Dir.)

Explorer la ville contemporaine par les transferts. - Lyon, Presses Universitaires de Lyon, 2017. $-221 \mathrm{p}$.

ISBN : 978-2-7297-0917-4

Les huit chapitres publiés sous la direction des deux chercheures renommées en sciences sociales et politiques exposent les résultats de différentes recherches conduites sur la ville contemporaine, analysée sous l'aspect de sa forme physique, puis comme objet des procédures de l'intervention publique et des promotions immobilières, enfin comme lieu des sociabilités où les habitant-e.s, les citoyen-ne-s, déploient leurs activités quotidiennes : commerces, temps libre, alimentation. En se différenciant d'un modèle unique de la ville globale, apparemment dominant, que rien ne peut arrêter caractérisé par la diffusion partout des mêmes messages publicitaires, des mêmes formes de ségrégation sociale, de pollution, par une élite internationale qui circule d'une ville à l'autre - les auteurs du volume insistent ici sur la diversité des architectures, l'originalité des modes de vie locaux, le poids de l'héritage historique (matériel et immatériel) : c'est-à-dire les phénomènes qui donnent encore aux villes contemporaines les formes les plus variées, originales, différenciées ${ }^{6}$.

La notion-clé, le fil d'Ariane, est celle de transferts, "que l'on entend ici comme la circulation de personnes, mots, choses, observés à travers ses motivations,

6 Cette note de lecture a été traduite de I'italien par Alain Tarrius. 
modalités, implications». Comme expliqué dans l'essai introductif, I'approche adoptée dans les différents cas exposés veut surtout se différencier de la méthode comparative qui est accusée, par les sociologues autant que par les historiens, de produire un catalogue d'analogies et différences par rapport à des configurations données, typologiques abstraites. Depuis les années 1990, au contraire, on a commencé à privilégier les dynamiques transnationales - leur convergence ou divergence - considérées décisives dans le procès de construction des faits intra nationaux. Dans ce nouveau courant d'études, l'analyse des politiques publiques et en particulier des politiques sociales transnationales joue un rôle important.

Au-delà des différents cas analysés, ce qui est intéressant est le fait d'avoir thématisé : a) l'élément des transferts (transferts comme mouvements de personnes, d'idées, de projets, de cultures) et b) le rôle, dans cette dynamique globale, d'un côté, du marché de la consommation (un marché de plus en plus international) et, de l'autre, des projets ou des personnes qui endossent une fonction de passeurs (du global au local, mais aussi l'inverse) dans des processus qui concernent la ville, soit dans ses quartiers, lieux de résidences familiaux, soit dans ses sites urbains d'échanges collectifs.

Les transferts sont ainsi considérés soit du point de vue d'échanges matériels (les flux commerciaux transnationaux véhiculés par les migrants décrits par Alain Tarrius, ou la transformation urbaine liée à l'installation de restaurants, dans le cas des Colombiens à Paris de Marcia A. Sierra), soit dans un sens d'échanges immatériels (la présence d' "experts européens» lobbies - regroupés près des ministères, et des associations d'habitants locaux). Autre thème, celui de la reproduction internationale des formes urbaines les plus contemporaines - comme, par exemple, les «ensembles résidentiels fermés/ spécialisés " que précisément ce genre de construction a favorisé. C'est là le cas examiné par Eleonora Elguezabal (les torres country à Buenos Aires) qui analyse d'une part les conflits et les conséquences sociales du cas considéré, et d'autre part le dialogue qui s'instaure entre les universitaires argentins et les experts nord-américains dans le débat international en cours sur la «privatisation » des espaces.

Un processus de sens contraire du transnational au local - est celui analysé par Éric Cheynis au Maroc dans le contexte urbain et péri-urbain : ici la clé de la transformation de la ville, réalisée par la politique de "lutte contre la pauvreté", réside dans le renouvellement des élites, avec la naissance d'un groupe de spécialistes, mais surtout par la valorisation que les associations locales ont reçue d'un nouvel espace professionnel formé d'intermédiaires légitimés par l'expertise internationale. Les cas d'appropriation' et de réutilisation (réelle et symbolique) des maisons bâties en Algérie par les citoyens émigrés et leurs fils (Jennifer Bidet) présentent un intérêt majeur dans ces problématiques. Clément Barbier, dans son exposé sur le "développement intégré des quartiers" à Hambourg (1990-2010), insiste sur le rapport entre politiques publiques et participation des groupes locaux. La recherche prend en considération la genèse de ce rapport, avec, d'un côté, la mobilisation des ressources des ministères locaux et, de l'autre, la participation des associations et des groupes d'habitants, avec la présence d'experts européens, tous convergeant sur l'opérativité de la catégorie, typiquement européenne, de "quartier à problèmes ". La participation est la clé de voute de la recherche conduite, avec une approche historique, par Cécile Cuny : I'objet est formé par les tentatives d'intervention de requalification sociale dans le grand quartier berlinois de Marzahan, bâti à Berlin-Est dans les années 1970, et profondément marqué par sa double vie, avant et après la chute du Mur. Une approche sociohistorique caractérise aussi la recherche sur la réappropriation par les nouvelles 
générations d'habitants de la Maison radieuse de Rezé de Le Corbusier, avec la réutilisation des espaces collectifs (Sabrina Bresson).

Dans l'ensemble, le volume représente, comme l'indique la synthèse des deux éditrices, une tentative réussie de marquer un pas en avant par rapport aux thèmes récurrents de la planification et de la réforme des ensembles résidentiels populaires, qui ont longtemps caractérisé la sociologie urbaine, mais aussi par rapport à une sociologie urbaine qui a surtout illustré les étapes vers un modèle unique de ville globale et uniforme. L'ensemble des cas ici examinés prouve que les diversités, les spécificités, sont aussi importantes que les généralisations mondiales et fondent l'originalité de la vie de la ville et de ses habitants, d'un rapport productif de conscience du fait local; cela est encore tributaire d'un autre phénomène qui semble accompagner la globalisation : la diffusion des nonlieux commerciaux et collectifs souvent répulsifs. C'est le signe que chaque noyau d'habitants veut imprimer ses singularités dans la ville où il vit (ou a vécu), qu'il s'agisse de familles de migrants agrégés en milieux urbains, ou encore d'habitants transférés solidairement dans des quartiers anonymes : ce sont les cas ici considérés par Jennifer Bidet (les maisons bâties par les émigrés en Algérie) ou par Marcia A. Sierra (la restauration colombienne à Paris), et, pour le second volet, la participation des habitants aux tentatives de réforme à Marzahn (Cécile Cuny), ou l'exploitation des petits jardins dans la Maison radieuse (Sabrina Bresson).

En conclusion, le besoin de marquer physiquement la présence de sa propre vie dans le monde urbain tend à augmenter chez les habitants au fur et à mesure que croît l'anonymat transmis par les grandes villes et la stéréotypie de la globalisation. Ce d'autant plus là où les villes possèdent un passé - loin ou récent - riche d'histoire, de vie sociale et pas seulement de biens à consommer (ce sont les cas ici considérés de Berlin et de Paris). Dans ces dernières villes typiquement européennes, dirait-on, l'on ressent encore plus l'exclusion et ce sentiment peut s'accompagner d'un besoin de réaction, sur le mode d'une recherche de compensations : en Italie c'est le cas de quartiers comme le Corviale à Rome et les Vele à Naples.

Mariuccia Salvati Professeure d'histoire contemporaine Université de Bologne

\section{Nouss, Alexis \\ La condition de l'exilé. - Éditions de la Maison des sciences de l'homme, Paris, 2015. - $176 \mathrm{p}$. \\ ISBN : 978-2-7351-2040-6}

Alexis Nouss, professeur de littérature générale et comparée, publie un essai dans lequel il se propose de dépasser les informations qui caractérisent la catégorie des migrants, chiffres, statistiques, politique de gestion, cela pour définir la "condition exilique", montrer la diversité des parcours individuels et collectifs, dégager le noyau existentiel commun à toutes les mobilités humaines.

L'auteur nomme exiliance ce noyau existentiel. Cerner cette réalité met en évidence l'expérience subjective du migrant et fait comprendre l'identité nouvelle qu'il acquiert durant son déplacement. Alexis Nouss souligne que tout exil amène une mutation de l'individu. Celui-ci passe d'un lieu à un autre et perd la conscience d'une appartenance entière à I'un de ceux-ci. Ce traumatisme plus ou moins fort et plus ou moins conscient constitue une mise en question et une tension de l'être. L'exil géographique de double ainsi d'un exil intérieur, d'une sorte de déchirure psychique qui ébranle les certitudes intimes. La langue et la narration du parcours de vie illustrent le partage : le sujet se trouve pris entre deux idiomes, deux rhétoriques, deux codes culturels 
qui ne lui permettent plus de s'identifier pleinement aux repères du lieu d'origine ni d'adopter ceux du lieu d'arrivée.

La démonstration est illustrée par des exemples empruntés aux religions et à la littérature : l'exode des Hébreux, la fuite en Égypte, I'Hégire, Ovide, Dante, Byron, Victor Hugo, Rimbaud, Carlo Levi et bien d'autres sont convoqués et leur expérience propre est analysée. Ainsi l'Othello de Shakespeare est élevé au rang de type : exilé, maure de Venise avant d'être mort à Venise, il est habité par un sentiment paranoïde, obsédé par la trahison, déchiré entre sa culture de naissance et les valeurs du lieu de résidence; inadapté au monde environnant, il cherche repère et réconfort. La mer, topos classique, symbole de l'exil, vraie patrie d'Ulysse, apparaît comme un espace menaçant, inconnu et en même temps une promesse de liberté. Aussi l'exil n'est-il pas toujours synonyme de drame et de traumatisme. À cet égard, l'auteur aurait pu insister davantage sur l'idée que les croyants se font de la condition humaine: I'homme ne serait qu'un migrant, exilé sur la terre et cherchant le chemin menant à sa vraie patrie située vers l'au-delà.

L'auteur ouvre des perspectives intéressantes sur les "non-lieux» d'exil, ces villes que les écrivains désignent par une simple initiale, ces logis figés et anonymes brossés par les peintres, ces espaces vides chers aux surréalistes. Dans un registre plus concret, les non-lieux peuvent s'incarner dans les centres de rétention, zones de regroupement, camps de transit et autres lieux d'enfermement qui ponctuent la planète. Là se retrouvent des individus "arrivés" sans pour autant "être parvenus à destination». Le non-lieu exilique sert parfois de porte d'entrée dans l'infini. Ainsi pourrait être évoquée l'expérience de Jean-Marie Le Clézio, errant sur le globe, mais s'arrêtant un instant au Cap de Nice : là, allongé sur les rochers blancs, percevant seulement le bleu du ciel et le bruit de la mer, emporté par une perception sensorielle intense, il pense entrer en communion avec l'infini. Alexis Nouss montre enfin une dimension inattendue : non l'individu s'exilant d'un lieu, mais le lieu abandonnant l'individu par déplacement de frontière, changement d'appartenance nationale, modification toponymique. II arrive que le retour au pays natal se révèle impossible, soit que le pays ait changé, soit que l'individu ait changé : «J'ai deux amours, mon pays et Paris", chantait Joséphine Baker.

Alexis Nouss plaide in fine pour un remplacement du droit d'asile classique répondant imparfaitement aux droits de la personne en ce qu'il ne reconnaît pas le droit d'émigrer et ne prend pas en compte les singularités, les différences, la condition d'exiliance engendrées par le déplacement.

Cet essai, nourri par de nombreuses références, exige une lecture attentive et pourrait alimenter la réflexion des décideurs qui, délaissant un moment les horizons chiffrés et les codes juridiques, s'aventureraient dans le monde des idées.

Ralph Schor

Historien, Professeur émérite Université de Nice-Sophia-Antipolis 